\title{
XXI.
}

\section{Ueber Grundabsicht und Entstehungszeit von Platons Gorgias.}

\author{
Ton \\ Prof. $\mathbf{P}$. Natorp in Marburg.
}

Ueber Gehalt und Anlage des Gorgias in aller Kürze etwas zu sagen, was der Grösse des Gegenstandes angemessen ist, muss recht schwer sein; auch ist der Eindruck der Schrift an sich ein so klarer und mächtiger, dass für denjenigen, der sich ihm nur ungetheilt, ohne viel Klügeln hingibt, eigentlich kein Wort mehr darüber zu sagen nöthig sein sollte. Doch ist es unerlässlich, die Grundabsicht der Schrift auf einen möglichst präcisen Ausdruck zu bringen, um auf dieser Basis, wenn möglich, über ihre Stellung im Ganzen der philosophischen Wirksamkeit Platons Klarheit zu gewinnen.

Nach dem ersten Anschein nimmt der Gorgias mit den Rhetoren den Kampf auf, wie der Protagoras mit den Sophisten. Doch erkennt man bald aus der Art, wie der Kampf geführt wird, dass der Angriff weit ernstlicher den Staatsmännern gilt. Noch ein wenig schüchtern weist Gorgias, schon offenherziger Polos auf die durch das Mittel der Redekunst zu erlangende Macht im Staate; vollends die Auseinandersetzung mit dem Staatsmann Kallikles fasst das Problem erst bei seiner wahren Wurzel und bringt den Gegensatz der Anschauungen, der hier geschildert werden sollte, zum allerschärfsten Ausdruck. Doch anch damit ist der Gehalt des Dialogs nicht erschöpft. In letzter Linie vielmehr handelt es sich um die wahre Eudämonie, um die rechte Lebens- 
führung; um die Reinigung der ethischen Grundansicht, die Scheidung von Wollen und Belieben, und damit des Guten von der Lust, durch welche das berückende Idol der Nacht zertrümmert und in seiner Nichtigkeit blossgestellt wird. Dem entspricht, dass als letztes, positirstes Resultat ethische Philosophie als Grundlage wahrer Statskuust zum Ersatz für das Verworfene angeboten wird. Die Bekämpfung des Standes der Rhetoren und Staatsmänner - was hier fast Eins ist - ist also schliesslich nur Aussenseite; Platon sieht darin nur den prägnantesten Ausdruck des blinden Machtstrebens der Zeit ${ }^{1}$ ); und auf dieses, auf die ganze Lebensrichtung von damals, insbesondere auf den politischen Zustand Athens zielt im Grunde die ganze Darlegung, die aus dieser überall durchschimmernden, einigemale auch recht schroff und nackt ausgesprochenen Oppositionsstimmung ihr tiefes, hinreissendes Pathos schöpft. Die Absage an den Zeitgeist wirkt hier, trotz des weit geringeren änsseren Apparats, weit mächtiger als etwa im Protagoras; clas Streiten wider den Sophistenwahn der Menschenerziehung will uns fast etwas jugendlich anmuthen gegen diesen neuen Kampf, der die ganze, gesammelte Kraft des gereiften Mannes forderte. Nirgends hat Platon. sein in der Apologie gegebenes Versprechen grossartiger erfüllt ${ }^{2}$ ).

Eine Absage an den Zeitgeist nannte ich die Schrift: das ist viel zu wenig gesagt. Gerade die Vergleichung mit den sokratisirenden Gesprächen muss es fühlbar machen, wie sehr hier der Standpunkt einer bloss negativen Kritik verlassen ist; wie Platon sozusagren Alles daran setzt, zu eiver centralen, für immer festen Stellung in der entscheidendsten aller Fragen, nämlich der des sittlichen, vorzudringen, und damit zugleich die sichere Grundlage

1) Daher wird die Rhetorik kaum weiter geprüft als eben, sofern sie als Waffe im Wettbewerb um die Staatsgewalt gilt und von ihreu Vertretern selbst angepriesen wird: sodass für eine die Redekunst an und für sich betreffende Erïrterung (Phädrus) Stoff genug übrig bleibt. (Vgl. unten Anm. 3.)

3) Apol. 39 (I). Bis Jemand entscheideude Gegengründe lringt, werde

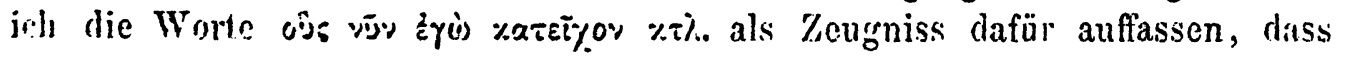
l'latou ror der Apologie wohl überhaupt nicht als Schriftsteller, sicherlich nicht mit schriften, welche eine Kritik des öfentlichen Zustands von Athen entbielten (\%. B. Protagoras), aufgetreten ist. 
für ein positives Wirken im Staate (auf dem indirecten Wege der philosophischen Erziehung) zu gewinnen. Gewiss sind die ethischen Grundïberzeugungen, die hier entwickelt werden, keine anderen als die sokratischen, wie man sie schon aus der Apologic und dem Kriton erkennt. Doch aber, welcher Unterschied in der Art und Tiefe der Begründung, in der Sicherheit des Bewusstseins, in der Energie der Anssprache. Schon die Anlage des Gesprächs zeigt den gewaltigen Fortschritt; diese klare Einheit, dieser feste Zusammenschluss bei so reichem und mächtigem Inhalt, diese Dentlichkeit und wie naturgesetzliche Nothwendigkeit der Gliederung in der auf fortschreitender Verinnerlichung des Problems beruhenden Steigerung von Gorgias zu Polos zu Kallikles, mit dem Abschluss in dem ewigwahren Mythos des Todtengerichts. Noch grösser fast wirkt diese ganz dichterische Gestaltungskraft; diese einzige Fähigkeit in die Seele des Anderen sich hineinzudenken, den äussersten Gegensatz kler eiguen Lebensanschauung in einem Typus - oder um denn ganz platonisch zu sprechen, einem "Paradeigma“ hinzustellen, dessen Lebenswahrheit jedes Zeitalter der Menschengeschichte bestätigt; und dann wieder diese Macht des Dialogs, dies zur Rede stellen, diese Gewalt, das eigne Bewusstsein des Mitunterredners (und damit des Lesers) zum Zeugen aufzurufen; nicht mehr nur, im sokratischen Sinne, ihn zum Geständniss zu bringen, dass er mit sich selber nicht einig, in sich selber nicht klar ist, sondern in jenem positiveren, plato-

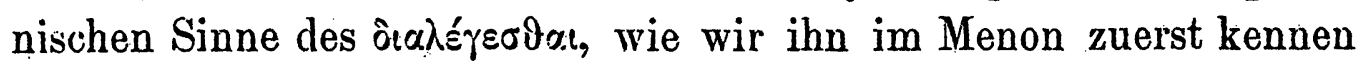
lernen, die Erkenntniss des Wahren ihn im Grunde der eignen Seele wiederaufspüren und sich darauf wiederbesinnen zu machen.

Das hatte ich im Gedanken, wenn ich meinte, es sei zu wenig gesagt, dass der Gorgias eine Absage an den Zeitgeist enthalte. Es ist nicht mehr eine Absage, es ist ein Ringen mit dem Zeitgeist, ein Ringen wie auf Leben und Tod. Dieser auflodernde Zorn, er wurzelt in unzerstörlicher Liebe: in jener tapferen Liebe des Arztes, der das Schneiden und Brenuen nicht scheut, denn er weiss, es ist zum Heile.

Das muss man herausfühlen, ich sage nicht, um den Gorgias zu geniessen, sondern um seine Bedeutung sich klar zu machen, 
und danach denn auch seine Stellung im Ganzen des platonischen Wirkens zu begreifen. Das ist es ja wohl, wodurch Platon, in praktischer Absicht, über Sokrates hinausgeht: dass er sich positiv die Aufgabe stellt, seine Zeit umzuwandeln, auf sie zu wirken in Kraft der Ueberzeugungen, welche Sokrates in ihm zum Leben erweckt, deren Macht er án sich zuerst erprobt hat, und in denen er die alleinige Rettung sieht für sein Volk, für seine Zeit. Es ist in der That meine Ueberzeugung: dass im Gorgias der Plan des platonischen Wirkens, wach ethisch-politischer Seite, niedergelegt ist; derselbe Plan, der seine genauere Ausführung gefunden hat - im Staat.

Daraus ergäbe sich nun schon ein, wie mir scheint, ziemlich sicherer Schluss hinsichtlich der Stellung des Gesprächs in der ganzen Reihe der platonischen Schriften: dasselbe ist an das Ende der Schriften von sokratischem Charakter, oder richtiger an den Beginn der specifisch platonischen Wirksamkeit zu setzen; d. h. es folgt, nicht bloss auf Apologie und Kriton, sondern gleichfalls auf Protagoraș und die drei mit diesem eng verknüpften Gespräche Laches, Charmides, Menon; es geht vorher allen sonstigen Schriften von nicht sokratischem Charakter.

Nach Schleiermacher hätten wir im Phädrus das Programm der philosophischen Wirksamkeit Platons und ebendeshalb seine frühste Schrift $z u$ sehen. Ich kann dem nicht beitreten, nicht bloss aus dem Grunde, der für die Mehrzahl der Forscher bestimmend gewesen ist: dass das eigenthümlich platonische Wirken die sokratisirende Periode nicht nur voraussetzt, sondern - wie gerade der Gorgias bestätigt - bewusst überwindet; vielmehr auch, wenn man diesen Fehler berichtigt und also von den sokratisirenden Gesprächen absieht, so ist selbst dann jene Ansicht nur halb wahr: das Programm des platonischen Wirkens enthält an erster Stelle der Gorgias, und nur in ergänzender Weise der Phädrus. Dass der letztere bloss die Form der Philosophie behandelt, betout Schleiermacher selbst; eben deswegen enthält el Platons Programm nur zur Hälfte; eben deswegen fordert er eine Ergänzung, wie nur der Gorgias sie bietet. Aber der Phädrus ergänzt vielmehr den Gorgias, nicht der Gorgias den Phädrus; wie 
eine durchgängige Vergleichung beider Schriften es, wie ich glaube, \%ur Evidenz bringt ${ }^{3}$ ).

Richtiger hat Schlciermacher erkannt, dass zwischen Gorgias und Theatet selrr bestimmto Bezichungen obwalten, welche vielleicht nöthigen, jedenfalls empfehlen, sie auch zeitlich nahe ancinander $\%$ rücken. Doch bestehen nicht minder genaue Beziehungen \%wischen Gorgias und Phädrus, und zwischen Phädrus and Theïtet. Dass die vier Schriften: Menon, Gorgias, Phädrus, Theätet zusammengehören und annähernd dasselbe Stadium der Entwicklung der platonischen Philosophie darstellen, hat $\mathrm{Z}$ eller richtig erkannt, der jetzt (Phil. d. Gr. IIa, 4. Aufl, S. 540-544) auch in der Anordnung dieser vier Gespräche nahezu die Auffassung vertritt, welche sich uns als die richtige ergeben wird.

Die vorherrschende Ansicht scheint dagegen $\mathrm{zu}$ sein, dass der Gorgias zu den Schriften von sokratischem Charakter zu zählen und deshalb, sowie namentlich wegen der vielen und bedeutungsvollen Hinweise auf den Tod des Sokrates, diesem, also dem Jahre 399 möglichst nahe zu rücken sei. Ich kann dem schon deshalb nicht beistimmen, weil mir scheint, dass der Gorgias weit weniger eine zweite Apologie des Sokrates, als (um Schleiermachers nur etwas zu schroffen Ausdruck einstweilen zu gebrauchen) eine Apologie Platons enthält. Ich meine, es sei evident, dass der Autor sich gegen Vorwürfe, die wider ihn selbst - natürlich als Sokratiker - erhoben worden sind, vertheidigt. 'Tor allem die wohlmeinende Ermahnung des Kallikles an Sokrates zur Betheiligung am Staatsleben und was darauf entgeguet wird, kann sich nur auf Platon beziehen, wie im allgemeinen ja auch anerkannt wird. Dann aber kann ich mir die Schrift nur in einem gewissen Abstand vom Tode des Sokrates denken.

Der Einzige unter den Neuern, bei dem ich fassbare Argumente für die frühere Ansetzung finde, ist v. Wilamowitz (Philol. Lnters. I $213 \mathrm{ff}$.). Derselbe beobachtet richtig, dass im Gorgias gewiss nicht absichtslos Archelaos von Makedonien als Typus des

3) Dass Phädr. 260 D sich auf den Gorgias zurückbezieht, halte ich für sicher; vgl. oben Anm. 1. 
T'ngerechten, der der Strafe entronnen, dem Sokrates, der sie schuldlos leiden musste, gegenübergehalten wird; so noch zum Schluss in der Darstellung des Todtengerichts (525 D, 526 C). Nun starb Archelaos in eben dem Jahre, wo Sokrates den Schirling trank; war dies dem Leser gegenwärtig, so musste jener Contrast desto eindringlicher wirken. Also, meint v. W., müsse die Schrift möglichst bald nach 399 verfasst sein; schon einige Jahre später wäre diese Gegenüberstellung minder wirksam gewesen. - Ich kann mich nicht überzeugen, dass ein Unterschied ron wenigen Jahren hier sehr ins Gewicht fiele. Das Beispiel lag an sich nahe und hatte etwa 5 Jahre später gewiss noch dieselbe Ueberzeugungskraft. Einen sicheren Schluss hinsichtlich der Abfassungszeit würde ich auf jene an sich richtige, auch für das Verständniss der Schrift förderliche Bemerkung in keiner Weise zu bauen wagen. - Entscheidender wäre, wenn er sich aufrechthalten liesse, ein zweiter Grund, welchen v. W. vorbringt. Nümlich der Gorgias müsse verfasst sein von einem von A then $\mathrm{A} b$ wesenden. Doch mir scheint das Gegentheil sicher. Die Strafrede des Kallikles setzt zweifellos voraus, dass der, an den sie gerichtet ist, also Platon, nicht etwa. ron Athen sich fernhält, sondern in der Stadt lebt und nur rom Staatswesen sich vornehm zurückhält, um in der Verborgenheit mit einer kleinen Schaar von Genossen der Philosophie zu leben. $\mathrm{Ja}$, ich meine, es müssten Reibungen zwischen den Philosophen und Staatsmännern schon vorausgegangen sein, die wir uns am natürlichsten auf dem Boden Athens denken würden. Wer in Athen bätte sich wohl sonderlich darïber aufgehalten, wenn Platon weltvergessen bei den P.thagoreern in Unteritalien oder wer weiss wo sonst sich Forschens halber aufhielt? Nein, sonderu er war anwesend, und man empfand seine Anwesenheit; man verstand in seiner Zurïckhaltung die Verachtung des öflentlichen Zustands der Stadt; man ahnte auch wohl, dass der Kreis, der um ihn sich erst zu sammeln begann und den man für jetzt noch meinte mit Geringschätzung behandeln $z u$ dürfen, vielleicht einmal zu einer achtunggebietenden Macht anwachsen könne. Lässt denn der Spott des Kallikles, dass Sokrates, bei so trefflichen Anlagen, den Markt und das Centrum der Stadt, wo wahre Mannestüchtigkeit sich er- 
probe, meide und es vorziehe in einem Winkel versteckt mit drei odler vier Jünglingen flüsternd sein Leben zu verbringen - lässt dieser Spott sich denn anders als auf Platon, und anders als in eben erklärten Sinne deuten? Auf Sokrates würde er keineswegs passen, er mied ja nicht die Mitte der Stadt und schloss sich nicht in Conventikeln ab. - Aber die "kolossalen Wahrheiten" des Gorgias, insbesondere die "herben und ungerechten "Urtheile iiber die grossen Staatsmänner der Vorzeit Athens, meint v. W., hätte Platon nicht wagen dürfen, wenn er damals in Athen sich aufhielt. - Nun, ich muss gestehen, dass mir die gegentheilige Voraussetzung ein gut Theil des Eindrucks der Schrift zerstören würde. Es war gerade kein Heldenstück, aus sicherer Ferne die bittere Anklageschrift in die Stadt zu senden, sich als Arzt und Retter, als den Einzigen, der wahrhaft für das Heil des athenischen Staates wirke, die wahre Staatskunst betreibe (521 D), anzupreisen; vollends, auf das böse Wort, dass man ihn, wofern er die Redekünste verschmähe, wohl straflos werde ohrfeigen dürfen (486 C, $508 \mathrm{DE} ; 527 \mathrm{~A}$ ), ja auf die Drohung, dass ihm selbst leicht das Schicksal des Sokrates bevorstehen könne (521 C, vgl. Men. $94 \mathrm{E}$ ), Antworten zu ertheilen, wie wir sie $511 \mathrm{~B}, 521 \mathrm{CD}, 522 \mathrm{DE}$ lesen, wie, dass man ihn sehr gelassen werde sterben sehen, wäre es aus Ermangelung schmeichlerischer Redekünste. Selbst abgesehen von dem allen braucht man nur einmal auf die zahlreichen Wen-

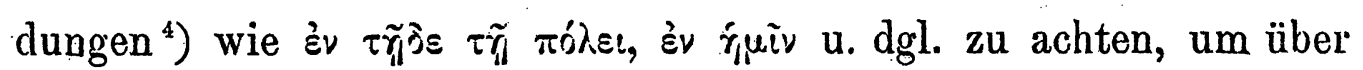
diesen Punkt, auch gegen das Urtheil eines Kenners der Zeitgeschichte wie v: Wilamowitz, vollständig beruhigt zu sein. Im Gegentheil wird nun dies Argument zu einer sehr wesentlichen Stütze unserer Auffassung. Ist der Gorgias sicher in Athen verfasst, so kann schon die allernächste Zeit nach Sokrates' Tode nicht mehr in Betracht kommen, da Platon eben diese unbestritten auswärts verbrachte. Der Gorgias kann aber auch nicht wohl diejenige Schrift gewesen sein, mit welcher sich Platon in Athen einführte; sie setzt den Ansatz wenigstens zur Schulbildung, sie setzt

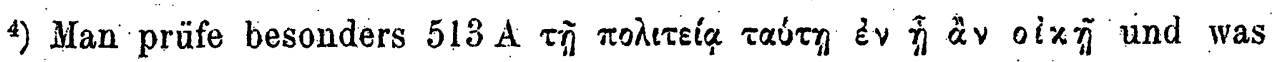

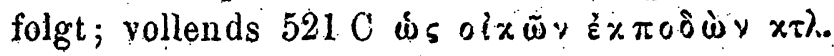


ein beträchtliches Ansehen, vorausgegangene sogar heftige Reibungen, wohl sicher auch schon erfolgte Angriffe auf frühere Schriften (wie Apologie und Protagoras) voraus; sodass wir sie jedenfalls um einige Jahre weiter werden hinabrïcken müssen.

Cebrigens kommt v. W. in die Verlegenheit, dass er z. B. den Protagoras weit später; in die Zeiten der Akademie zu setzen genöthigt wird. Ich weiss nicht, ob diese Annahme erst der Widerlegung bedarf, doch ist es nicht ohne methodisches Interesse, die Argumentationsweise zu prüfen, die zu solchem Schlusse führte. Ich lese (S. 218): „Der Mai 399 warf Platon aufs Krankenlager - und das Lachen hat er erst mehr als 10 Jahre später, als er in der Akademie lehrte, Protagoras, Euthydem, Symposion schrieb, wiedergefunden“. Und (219): „Das ist doch wahrlich kein modernes noch subjectives Empfinden, wenn man leugnet, dass der Phädrus in der Stimmung des Gorgias und Menon entstanden sein könne." Ich weiss nicht, ob man mit solchem Argument bei andern Autoren etwas ausrichtet; auf Platon scheint es mir unanwendbar. Platon schöpft seine Stimmung aus dem Gegenstande, nicht lässt er seine Gegenstände sich dictiren von der Stimmung; die ihn gerade beherrscht. Der Unterschied des Gegenstands erklärt den Stimmungsunterschied zwischen Menon und Gorgias; und so auch wohl zwischen Gorgias und Phädrus. Schon die Apologie zeigt übrigens keine ausschliesslich trübe, keine resignirte, sondern eine höchst kampfbereite Stimmung; vollends der Ton des Gorgias ist nicht bloss muthig entschlossen, sondern siegesgewiss, und in seinem unerbittlichen Hohne so überlegen, wie ich es nicht begriffe, wenn der Autor in freiwilliger Verbannung mit unthätiger Resignation dem Verderben der Stadt von weitem zusah. Gewiss ist der Phïdrus nicht in derselben Stimmung geschrieben; ihn trübt (sagt v. W.) nirgend ein Ton, der auf das Ende des Sokrates deutete. Er kann aber darum doch nach 399, er kann nach dem Gorgias, sogar unmittelbar nach ihm verfasst sein. Warum sollen wir dem Platon, nachdem er in sieben Schriften, deren keine es an Herbheiten fehlen lässt, die letzte sie bis zum äussersten steigert, seinc philosophischen Consequenzen aus dem Ereignisse des Mai 399 gezogen, nicht endlich gestatten, auf den Flügeln der Idee 
zu jenen Inseln der Seligen zu kurzer Rast zu flüchten, welche der Schluss des Gorgias dem Philosophen, der dem Welttreiben cntrounen ist, verheisst? - Erst der Erfolg in der Akademie soll Platons Stimmung rerbessert haben.' Ioh würde dergleichen nie zu behaupten wagen; übrigens setzt der Gorgias etwas wie eine Schule Platons voraus; desgleichen sicher der Theätet, an dessen Eutstehung noch in der zweiten Hälfte des ersten Jahrzehnts nach Zellers Beweisführung (Abh. d. Berl. Akad. 1886) nicht mehr zu rütteln sein dürfte; es stände also nichts im Wege, den Stimmungsumschlag, den man zur Erklärung des Phädrus für nothwendig hält, schou bald nach dem Gorgias eintreten zu lassen. Ich möchte, wie gesagt, auf solche Gründe keineswegs etwas bauen; doch wer sie nöthig hat, dem werden sie nicht fehlen. Allgemein habe ich Bedenken dagegen, auf Annahmen über den Lebensgang Platons andere über die Reihenfolge seiner Schriften zu stützen - zumal ohne die ernstlichste Berücksichtigung ihres inhaltlichen Verhältnisses.

Und so möchte ich auch für meine Ansetzung des Gorgias mich bloss hülfsweise auf die Lage des Autors, die er voraussetzt, berufen. In der That gelangt man zu demselben Schluss auf dem geradesten und sichersten, obzwar altmodischen Wege, indem man sich klar macht, dass der Gorgias, seinem Inhalt nach, die ganze sokratisirende Periode (d. h. die Schriften von der Apologie bis zum Menon) voraussetzt. Das hat man auch früher meist angenommen; aber sich die daraus zu ziehende Consequenz verborgen, indem man Protagoras, Laches, Charmides und wohl gar Menon noch zu Sokrates' Lebzeiten abgefasst sein liess; was für den letzteren schon wegen der Bezugnahme auf das spätere Schicksal des Anytos (35 A) unmöglich ist, aber auch für die drei anderen Gespräche Niemand zugeben wird, der sich deren Verhältniss zur Apologie ernstlich klar gemacht hat; erinnert sei für jetzt (ausser dem oben Anm. 2 Bemerkten) nur noch daran, dass nicht bloss Men. $91 \mathrm{CE}$, sondern auch Lach. $186 \mathrm{~B}$ auf den gegen Sokrates (in Verwechslung desselben mit den Sophisten) erhobenen Vorwurf, dass er "die Jugend verderbe", sich deutlich bezieht. Doch scheint es heute fast nöthiger erst das Verhältniss des Gorgias zu den ge- 
nannten Schriften ausser Zweifel zu stellen; hat doch die spätere Jatirung des Menon gegenüber dem Gorgias noch kürzlich an Gomperz einen beredten Vertheidiger gefunden.

Schon das positive Auftreten Platons im Gorgias zeigt den entschieden fortgeschrittenen Standpunkt. Noch behauptet zwar Sokrates, nichts zu wissen von dem was er vorbringt (506 A, 509 A) - aber doch, dass noch Jeder, der es anders zu sagen versucht, sich lächerlich gemacht hat, weun er von Sokrates geprüft wurde (509 A, cf. $527 \mathrm{AB}$ ). Das ist nicht nur ein andrer Ton, als den z. B. der Protagoras anschlug, sondern es setzt frühere Darlegungen über die ethischen Grundfragen ersichtlich voraus, und wo sollten wir die suchen, weun nicht hauptsächlich im Protagoras, in zweiter Linie etwa im Menon? Und noch entschlossener erklärt Sokrates: was im Gespräche sich ergeben, das bleibe fest und wohl verwahrt mit eisernen und stählernen Gründen ${ }^{5}$ ); und nochmals: gegenüber so vielen Sätzen, die alle widerlegt wurden, ist dieser allein festgeblieben, uóvos củ̃os ìp dïrfen wir'getrost 'zur Richtschnur unseres Lebens wählen. Nun handelt es sich dabei eben um den Begriff der Tugend - wie in sämmtlichen sokratischen Dialogen. Jeder wird sich erinnern, wie gerade dieser Begriff dort beständig als noch nicht gefunden galt; so ausdrücklich im Protagoras, im Laches, und noch im Menon, dessen Schluss gerade die Beantwortung der Frage „Was ist Tugend?" als noch ausstehend bezeichnet. Insbesondere wurde die Tugend zwar stets zurückgeführt auf Erkenntniss, und zwar des Guten, was aber das Gute sei, wurde ernstlich noch gar nicht gefragt. Hier im Gorgias zum ersten Nale wird der Begriff des Guten untersucht und durch strengste Scheidung von der Lust wenigstens negativ, sodann aber auch positiv, allgemein als táhos, bestimmter als Gesetz und Ordnung (auch als siurs $503 \mathrm{E}$ ) erklärt. Wie wäre nach dem allen noch eine so aussichliesslich negative

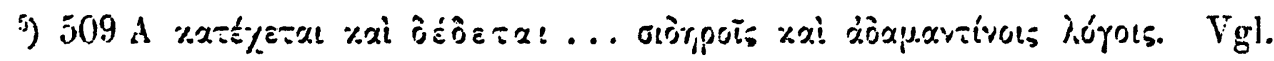

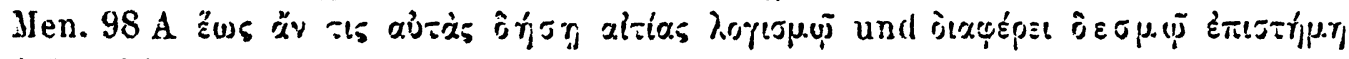

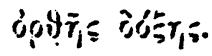

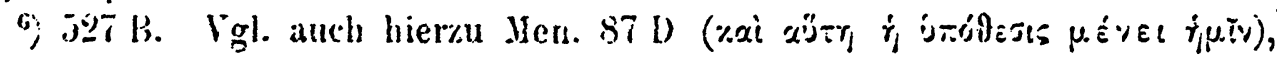

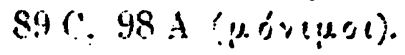


Behandlung derselben Begriffe möglich gewesen, wie in jenen vier Dialogen? War etwa, was hier nit eisernen und stählernen Gründen" festgemacht und zur Richtschnur des Lebens erhoben worden, hernach doch wieder zweifelhaft geworden? Ist nicht im Gegentheil die Grundansicht, die hier zuerst gewonnen wurde, fest geblieben im Staat, im Philebus? Gewiss künstlich wäre die Annahme, dass in irgendeiner Zeit zwischen dem Gorgias und diesen späteren ethischen Schriften Platon nochmals von der alten, sokratisch-negativen Behandlungsart dieser selben Grundbegriffe sollte Gebrauch gemacht haben.

Dies Argument, welches vielleicht für sich allein schon durchschlagend wäre, lässt sich übrigeus noch viel weiter ins Einzelne verfolgen. So erhalten wir im Gorgias nebenbei auch so bestimmte Erklärungen der einzelnen Tugendbegriffe wie ธwọposúvn, àvispsia u. s. w., wie man sie gleichfalls in jenen vier Gesprächen vergebens sucht. Man weiss, wie Protagoras, Laches, Charmides einen Sonderbegriff einzelner Tugenden fast auszuschliessen scheinen; wie in einer Stelle des Menon (88 A ff.) sogar Besonnenheit, Tapferkeit etc. nicht an und für sich Tugenden sein, sondern, wie andere "Güter", erst unter der Leitung der ópóvraıs zu Tugenden werden sollten, während sie daneben doch auch wieder als Einzeltugenden oder "Theile." der Tugend begegnen; sodass ihr wahres und positives Verhältniss zur Einen Tugend noch gänzlich unentschieden blieb. Nun haben zwar auch die Bestimmungen, welche der Gorgias gibt, später im Staat gewisse Modificationen erfahren; aber die Grundauffassung ist doch auffallend dieselbe geblieben, die Abweichungen sind mehr yon technischer Bedeutung. Auch hier also bestätigt sich, dass im Gorgias diejenigen Grundzüge der ethischen Ansicht erreicht sind, welche dem Platon dawernd festgeblieben sind.

Erwähnt wurde schon, worauf die scheinbare Ergebnisslosigkeit der vorgenannten vier Schriften gerade in ethischer Beziehung - wihrend sie doch von nichts als vom Begriff der Tugend handeln - zuletzt beruhte; sie beruhte darauf, dass die Tugend ausschliesslich unter dem sokratischen Gesichtspunkt der Erkenntniss behandelt wurde. Hier sind wirkliche Fortschritte zu verzeichnen, in der näheren Bestimmung des Begriffs derjenigen Erkenntniss, in 
der die Tugend bestehen sollte; es wurde erreicht, dass diese Erkenntniss, um es kurz zu sagen, die a priori-Erkenntniss des Einen, an sich und absolut Guten, nicht die empirische der mannigfachen relativen Güter des Lebens sein müsse. Aber der so dringlich geforderte a priori-Begriff „des" Guten wird, wie gesagt, nirgend erreicht oder nur ernstlich in Untersuchung gezogen. Im engsten Zusammenhang mit der Auffassung der Tugend als Erkenntniss wurde ferner die Frage nach der Lehrbarkeit der Tugend wieder und wieder aufgeworfen. Ihre Verneinung im Protagoras dürfte ernst zu nehmen sein: Sokrates musste sie, von seinem Standpunkte des Nichtwissens, verneinen; Platon bejaht sie erst im Menon, auf Grund der sicher ihm specifisch angehörigen, nicht sokratischen Lehre von der Anamnesis. Im Gorgias nun wird sowohl, dass Tugend ein Wissen, wie, dass ein Wissen nothwendig lehrbar sei, schlechthin vorausgesetzt, ohne dass der leiseste Zweifel daran auftauchte. So stimmt es auch allein zu der so ganz positiven Haltung des Dialogs. Dass übrigens diese selbstgewisse und entschiedene Haltung auf dem im Menon zuerst erreichten Resultate fusst, dafür bürgt die ständig wiederkehrende

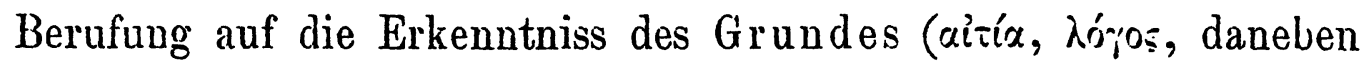
'óvos, besonders $465 \mathrm{~A}$ und 501A), durch welche im Menon (98 A) die Anamnesis geradezu definirt wurde. Wenn aber ferner nach diesem Kriterium die wissenschaftliche Erkenntniss von unwissenschaftlicher, grundloser Empirie unterschieden wird, so geht dies sogar über den Menon entschieden hinaus und sticht merkwürdig ab gegen die Unbestimmtheit, in welcher dort der Be-

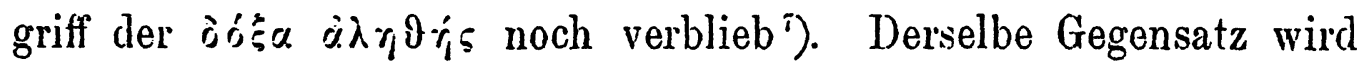

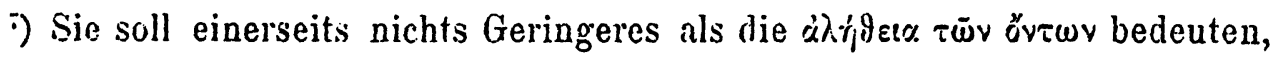
die von jeher in uns ist und nur noch zum Bewusstsein geweckt zu werden

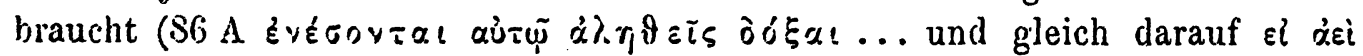

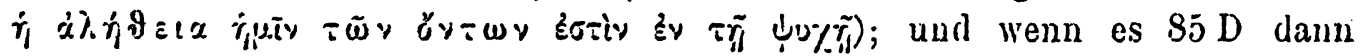
wieder heisst, dass wir die Erkenntniss aus uns selber schüpfen (àvaì $\beta \dot{\omega} y$

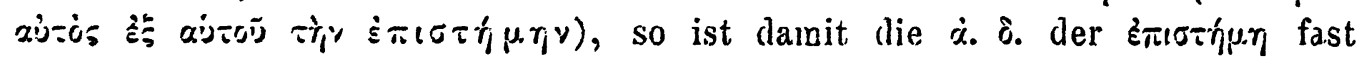

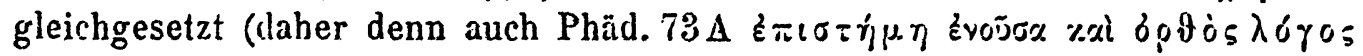
- nicht mehr i. oj $j_{5}^{\prime} \alpha$ ). Dagegen wird hernach sehr stark der Unterschied

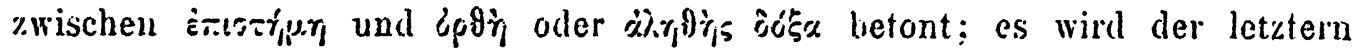
alle eständigkeit ahgesprochen, sie wird als blosser Schatten der Erkenntniss 
in Phïlrus ( $260 \mathrm{E}$ u. f., wo die Zurïckbezichung auf den Gorgias ron siebeck orkannt. jetzt auch von Zeller, II a ${ }^{4} 5+1^{1}$, anerkannt ist) in Erinnerung gebracht, im Theätet aus den tiefsten Tiefen der. bikenntnisstheorio abgeleitet; sodass auch hier der Gorgias über jene vier Schriften hinausgeht, dagegen mit solchen, die Jerler als specifisch platonische anerkennt, in eine Reihe tritt. Weiteres der Art würde eine speciellere Darlogung zu verzeichnen haben; das Gesagte wird genïgen, um den Fortschritt des Gorgias im allgemeinen zu kenn\%eichnen.

Es kommt nun aber cine ganze Reihe ron Momenten hinzu, die in frorgias entweder vollständig neu auftreten, oder höchstens mit dem Menon ihm gemeinsam sind, so aber, dass auch im letzteren Falle ein Fortschritt über den Menon hinaus unverkennbar ist.

Dahin gehört zuerst der nachdrückliche Hinweis auf Philosophie, unter diesem Namen. Man kennt die sehr allmähliche A usprägung dieses Terminus bis zu der prägnanten Bedeutung, die er zuerst bei Platon, und auch bei ihm nicht von Anfang an erhält.

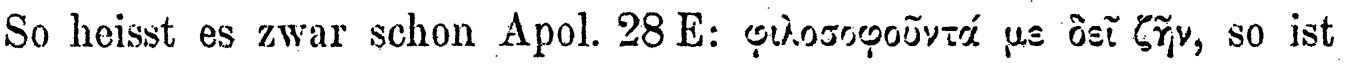
es die erste Frage des aus dem Feldzug zu seinem gewohnten

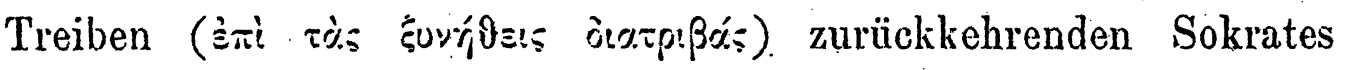

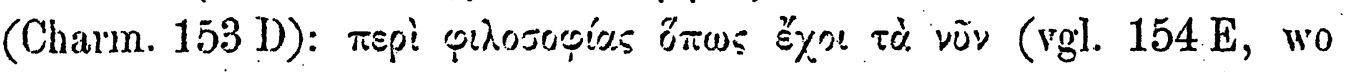
Kritias den Charmides als "weisheitsliebend" rühmt); aber keine dieser Stellen reicht entfernt an die Bestimmtheit heran, mit der Sokrates in Gorgias nicht nur Philosophie als seinen Beruf und

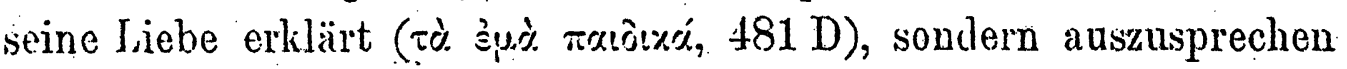

als dem. Wuhren gegenübcrgestellt. Wie nan das auch reimen möge, auf jeden Fall ergibt sich, dass der Begriff der òók $\alpha$ hier noch sehr im Ungewissen schwebt. Es fehlt namentlich die dem späteren platonischen Begriff der oós $x$ ganz wesentliche Beziehung auf das Gebiet (ler siunlichen Erfahrung; hicr scheint sie vielmebr die a priori-Erkenntniss (obwohl bloss ats óvapus) $\mathrm{zu}$ bezeichnen. Die wissenschaftliche Erkenntniss ist anf dem Grunde des

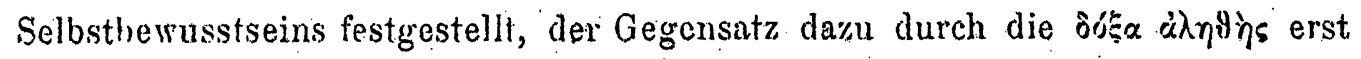
sehr unzureichend, gewissermassen provisorisch bezeichnet. Man mag daraus zugleich ersehen, wie ummüglich es ist den Menon auf den The ätet erst folgen zu lassen, in ihm wohl gar die Auflösung der Schwierigkeiten zu

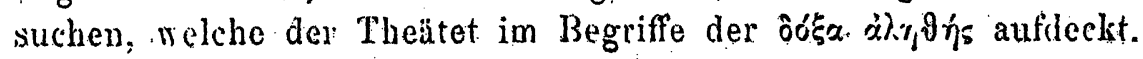


wagt: nicht ich, die Philosophie sagt so (482 A), und sie sagt

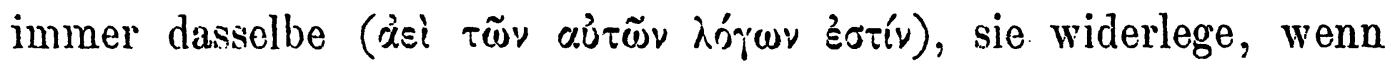
du kannst; worauf denn Kallikles (484 C, 485 A-D, 486 A cf. 487 C) mit der schönen Lehre antwortet, dass man es mit der Philosophie nur ja nicht zu weit treiben müsse. So wird denn durchgängig das Leben des Staatsmanns dem des Philosophen gegenübergestellt, be-

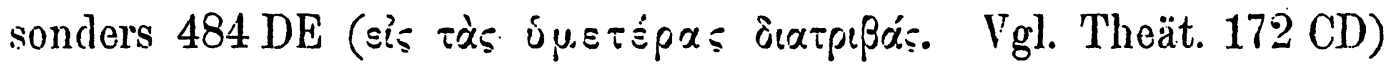

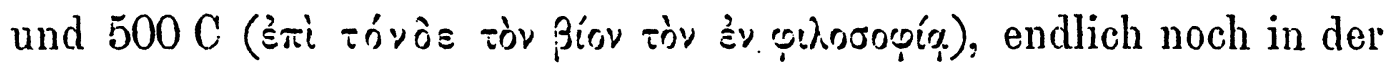
Darstellung des Todtengerichts ( $526 \mathrm{C}$ gegen $525 \mathrm{D}$ ). Fin so positiver Begriff ron Philosophie als festgegründeter Wissenschatt, deren Entscheidung gleichbedentend ist mit dem Worte der Wahrheit, war auf sokratischem Standpunkt unmöglich. Ich glaube darum nicht (mit v. Wilamowitz), dass der Phädrus, in welchem pihrorscíx. als Terminus begründet $z u$ werden scheint (278 D), dem Gorgias rorausgegangen sein müsse; wohl aber, dass die grundsätzlich negative Haltung, welche Platon als Sokratiker in den bisherigen Schriften noch einnahm, bewusst überwunden sein musste, beror von sidosrerín in solch positivem Sinne die Rede sein konnte.

Sodann finden sich zuerst im Menon Hindeutungen auf gewisse Interna platonischer Philosophie, welche als dem Leser nicht ohne weiteres bekannt und zugänglich vorausgesetzt, daher, nach einem naheliegenden Vergleich, geradezu als y.ustripa bezeichnet werden (Mcn. $76 \mathrm{~F}$ ). Darauf weisen auch hier bestimmte Anspielungen (493 B, $497 \mathrm{C}$ ), sn namentlich die Andeutung von der "geometrischen Gleichheit" (508 A), wo es sehr bezeichnend ist, wie Sokrates sofort abbricht, weil ja ein Kallikles sich um Geometrie nicht kümmere. Die Hervorhebung der Geometrie, schon im Menon so auftiillig ( $76 \mathrm{AE}, 86 \mathrm{E}$ u. bes. $82 \mathrm{Cfl}, 85 \mathrm{E}$ ), ist hier doppelt motivirt: imnerlich durch die Ausdehnung der ethischen Begriffe ron Ordnung und Cieset\% auf das Weltall als Kosmos, und zugleich ïuserlich durch dis sehr entschiedene Anlehnung an pythagoreische Anschauungen, sowohl $493 \mathrm{~A}$ (rgl. Böckh, Philolaos

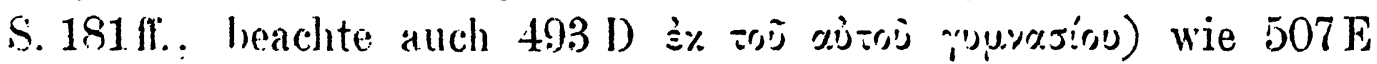
(wo sich Platon gerade lïr die Bezeichnung des Weltganzen als

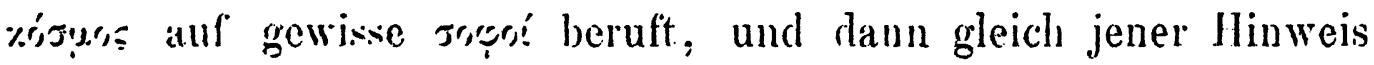
auf dic (jeometric folgt). Wir befinden uns in ganz pythagoreischem 
Zusammenhang. Auch der Mythos am Schluss hat damit Verbindung, wie die Andeutung der Unsterblichkeit (492E-493A, neben $523 \mathrm{~A}, 524 \mathrm{~B}$ ) und das Wort vom Hades als dem jizıòśs (49: B, vgl. $522 \mathrm{E}$ ff.) lehrt. Ganz besonders ist aber hier, neben der kosmischen Bedeutung der ethischen Grundbegriffe, die ausdrückliche Annahme eines unsichtbaren, übersinnlichen, unkörporlichen Reiches zn betonen: die Seele wird mit dem Tode vom Körper und den Sinnen entkleidet (523 DE); was über das im Menon Angedentete bereits weit hinausgeht, dagegen sehr geeignet ist, die ganz überschwängliche Darstellung des übersinnlichen Reichs im Phädrus vorzubereiten. Dass Platon damit den sokratischen Standpunkt weit hinter sich lässt - man erinnere sicb nur der durchaus zweifelnden Haltung des Sokrates in der Apologie hinsichtlich der Frage der Unsterblichkeit - bedarf keines Beweises. Aber es ist wohl mehr als blosse Vermuthung, dass hier auch die Ideenlehre schon in Hintergrunde steht. Legt

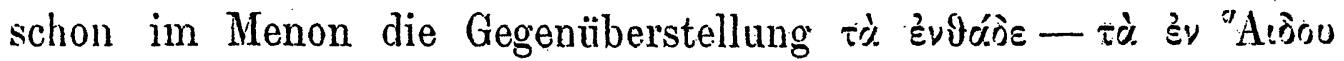

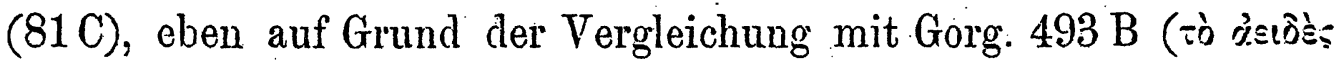
òं $\lambda \hat{s}^{\prime}(\omega \nu)$, eine solche Vermuthung sehr nahe (vgl. Ribbing, Die platonische Ideenlehre, I, $173 \mathrm{ff}$.), so haben wir im Gorgias ausser dieser Gleichsetzung (durch welche auch die Schilderung des Todtengerichts erst in die richtige Beleuchtung gerückt wird) auch directere

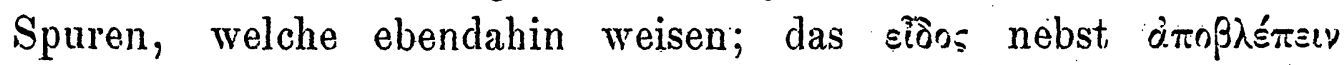
(503 DE; vgl. Men. 72 C) kommt dem entwickelten platonischen

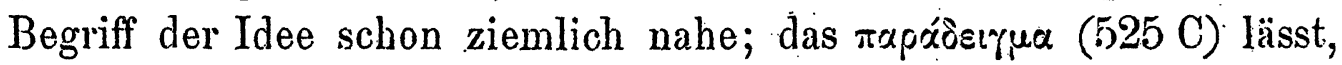
wenn es doch eben um ein Ewiges, Unsichtbares, Unkörperliches sich handelt, kaum eine andere Deutung za (vgl. namentlich

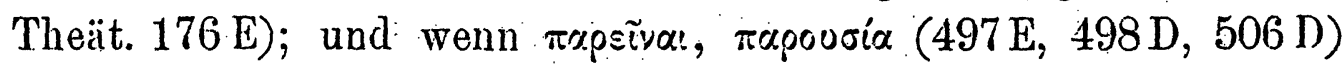
weniger entschieden im Sinne der fertigen Ideenlehre gebraucht scheint $^{8}$ ), so könnte das auf der absichtlich exoterischen. Behand-

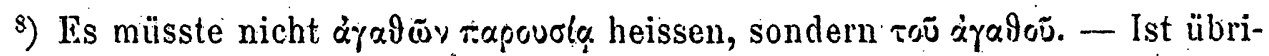
gens Euthyd. 301 A im Sinne Zeller's (Ph. d. Gr. II a $296^{2}, 531^{2}$ ) zu verstehen, so sieht man sich nach einer Stelle um, wo der Terminus früher von Platon gebraucht wäre. Ich finde - wenn vom Hipp. mai. abzusehen sein sollte -

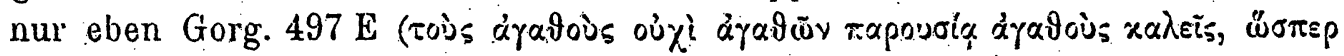

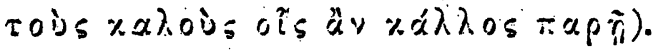


lung dieses Punktes beruhen. Denn wenn darauf im Plädrus die erste deutliche Aussprache der Ideenlehre eingeleitet wird mit den

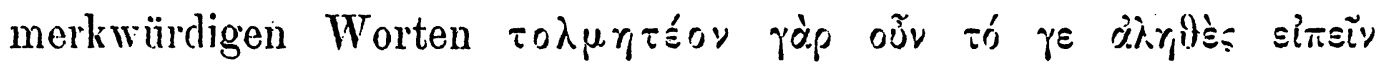
(247 C), so lautet das doch, als hätte er mit der schon erkannten Wahrheit bis dahin absichtlich zurr̈ckgehalten. Eine solche bewusste Unterscheidung exoterischer und esoterischer Behandlung nöthigt ja auch anzunehmen, was wir von den "Mysterien" und der geometrischen Erkenntiniss hörten; die Annahme ist um so leichter, wenn ein Schülerkreis bereits rorausgesetzt werden darf. I)ann aber wird durch diese Beziehungen zweierlei zugleich bewiesen: erstens, dass Platon ron dem sokratisch-negativen Standpunkt, wie er im Protagoras, Laches, Charmides, und nicht mehr ausschliesslich zwar, aber doch noch in weitem Umfang im Menon herrscht, sich bereits ziemlich weit entfernt hat; und zweitens, dass er einen gewissen Anhang, einen festen Kreis von Mitforschenden schon gefunden hat; was, selbst unabhängig von jeder Annahme über die Abfassungszeit der Dialoge Prot. bis Men., nöthigen würde, den Gorgias frühstens um die Mitte des ersten Jahrzehnts zu setzen.

Alle angeführten Gründe sind nun zwar auch für die spätere Abfassung des Gorgias gegenüber dem Menon beweisend, doch sei, namentlich mit Rücksicht auf Gomperz, darüber noch Einiges besonders bemerkt. Da scheint mir nun zuerst die eigenthümliche Rolle beachtenswerth, welche dem Kallikles in unserm Dialog zuertheilt wird. Wie soll man die Bedeutung dieser merkwürdigen Figur erk]ären? Der extremste mögliche Gegensatz der eignen Gesinnung wird rorausgesetzt, damit, was selbst einem so gesinnten Gegner im sokratischen Gespräch abgerungen werden kann, endgültig fest bleibe. Das ist die, nicht hineingelegte, sondern ausgesprochene Absicht der Einführung dieser Figur; man scheint sich aber bisher nicht Rechenschaft darüber gegeben $z u$ haben, dass dadurch das Verfahren der vorigen Dialoge mit Bewusstsein verlassen und berichtigt ist.

Polos wirft (461 B) dem Gorgias vor, er hahe aus Scham dem Sokrates zugestanden, was er gar nicht zuzugestehen brauchte, und sich dadurch ron dem listigen Gegner in Widerspruch ver- 
wickoln lassen; Kallikles erhebt ( $482 \mathrm{C}$ fi.) genau denselben Vorwurf gegen Polos, wobei er ebenfalls das Lnrecht des sokratischen Verfahrens scharf rügt. Und wie antwortet Sokrates? (486 D bis $488 \mathrm{~B}$ :) Er habe in Kallikles den Prüfstein gefunden, an dem seine eigene Gesinnung ihre Kraft erproben könne, sodass, was selbst er; Kallikles, einzugestehen genöthigt werden könne, fortan als endgïltige Wahrheit feststehe ${ }^{9}$ ). In solchem Sinne lobt er, ironisch genug, den ellen Freimuth, die "Parhesie" dieses merkwürdigen Helden - während freilich am Schluss der Verhandlung (508 BC) sich herausstellt, dass, was Gorgias-Polos angeblich aus blosser Scham zugcstanden hatten, vielmehr eben die Wahrheit ist, zu deren Anerkenntniss auch Kallikles genöthigt ist.

Also deutlich wird uns gesagt: das Problem muss radicaler angefasst werden; der Beweis des Sittlichen darf nicht länger auf Zugeständnisse rechnen, die der Scham, nicht der inneren Ueberzeugung des Gegners abgezwungen werden.

Das war nun aber doch ganz ersichtlich das Verfahren, zuerst im Protagoras, dann im Menon ${ }^{10}$ ). Hier im Gorgias wird es an den beiden Figuren des Gorgias und Polos wiederholt und ausdrücklich bemerklich gemacht - um aber dann, eben durch die Aufstellung eines radicaleren Gegners in der Person des Kallikles; endgültig verbessert $z u$ werden. Es ist gewiss ein sicherer Schluss: dass keine platonische Schrift, welche das hier so ausdrücklich berichtigte V'erfahren noch unbefangen (zumal auf dieselben Probleme) anwendet, später als der Gorgias geschrieben sein kann. Da nun gerade das Verhalten des Menon dem des Gorgias und Polos (als deren Gesinnungsgenossen er sich eben auch darin zu erkennen gibt) ganz analog ist und das Gespräch eine tiefere Ergründung

9) Man beachte auch hier die starke Betonung des definitiven Charakters der hier $z u$ erreichenden Feststellungen: $486 \mathrm{E} \alpha \dot{u} \tau \dot{\alpha} \tau \dot{\alpha} \lambda \eta \vartheta \tilde{\eta}, 487 \mathrm{E} \tau \omega \overline{\text { oัv }}$

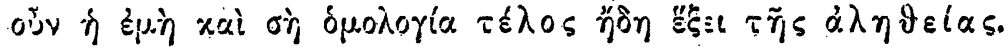

10) Es ist sehr merkwärdig und für das Bewusstsein, mit welchem Platon vorgeht, bezeichnend, dass der Gegner allemal gefasst wird auf Grund der Gleichsetrung des áyàóv mit dern $x \alpha \lambda$ óv, dessen schwankender Begriff die scharfe Grenze zwischen Scheinen und Sein verdeclit hält. (IIan prüfe Prot. $349 \mathrm{E}$, anch $352 \mathrm{CD}, 359 \mathrm{E}$; Jen. 77 B; Gorg. 474 C.) Kallikles muss den Solintes auf eben diesem Kunstgriff ertappen, Gorg. $482 \mathrm{DE}$. 
der sittlichen Begriffe eben deshalb nicht erreicht, weil er, immer auf das Schickliche bedacht, die Skepsis nicht weit genug treibt und sich zu bald ganz in die Bahn des Sokrates mitfortnehmen lässt: so ist es unmöglich, dass der Menon auf den Gorgias erst gefolgt sei; er müsste, nach ihm, als ein schwächliches Nachspiel erscheinen, während wir nun in ihm ein durchaus passendes Vorspiel zum Gorgias sehen und die nicht tiefer führende Behandlung des Ethischen uns daraus erklären dürfen, dass in dieser Richtung ein wesentlicher Fortschritt über Protagoras, Laches, Charmides hinaus noch nicht beabsichtigt ist, sondern fïr den Gorgias aufgespart bleibt, dagegen rorerst nur die erkenntnisstheoretische Frage, die durch jene Gespräche so nahegelegt war, einen bedeutenden Schritt weiter gefördert werden sollte.

Nun sieht freilich Gomperz (Plat. Aufs. I, 1887) einen Beweis für die spätere Abfassung des Menon gegenüber dem Gorgias in der Behandlung der Staatsmänner in beiden Dialogen. Vielleicht hätte das Problem von vornherein nicht so isolirt werden sollen: das Urtheil über die Staatsmänner ist in beiden Schriften nur der Ausfluss der ethischen Anschauungen, die sie entwickeln. Diese mussten zu allererst verglichen werden, wo sich denn wohl sofort klar herausgestellt hätte, dass der Standpunkt des Gorgias ein entwickelterer ist. Aber selbst unmittelbar musste einleuchten, dass der Gesichtspunkt bei der Beurtheilung der Staatsmänner hier und dort ein ganz verschiedener, und der verschiedene Ausfall des Urtheils nur die Folge dieser Verschiedenheit des Gesichtspunkts ist. Nämlich der Menon behandelt die Frage ganz im Zusammenhange des alten Problems der Lehrbarkeit der Tugend, allgemein ihres Verhältnisses zur Erkenntniss, daher wesentlich im Sinne des Protagoras und in möglichst ausdrücklicher Erinnerung an diesen "). Die Frage des sittlichen Verdienstes wird ernsthalt gar nicht erhoben; der Vorwurf, dass man die Staatsmänner schmähe, wird zurückgewieseu durch die Erinnerung, dass es sich jetzt darum gar nicht handle, ob ihr Wirken Lob oder Tadel ver-

iij Adulich uriheilt /aller, in Archiv f. Gesch. d. Philos. I 418 und jetkt Ph. d. (ir. Ilat istz". 
diene, sondern ob es auf Erkenntuiss berule uder nicht (93 AB). Jaher ist auch das diesen Hoclwweisen gespendete Lob genau so ironisch zu nehmen ${ }^{12}$ ) wie schon in Protagoras, oder wie im Menon selbst die Inschutznahne der Sophisten. (wo man z. B. die Betonung des Gelderwerbs, den sie ihrer Vortrefflichkeit danken, micht übersehen wird). Dass sie tüchtig gewesen, lässt sich Sokrates ron Anytos bejahen; er selbst will es damit gewiss nicht in jedem Sinne behauptet haben. Ernsthaft möchte ich wenigstens nur die allgemeine Anerkennung nehmen: dass es wahrhaft tüchtige Staatsmänner gibt und gab (Men. $93 \mathrm{~A}$ ); doch das erkennt fast ${ }^{13}$ ) mit denselben Worten auch der Gorgias an (526 A). Aber freilich fasst er die Frage weit schärfer im ethischen Sinne, und erklärt nach diesem Maassstab die Miltiades, Themistokles, Kimon, Perikles. gradezu für schlechte Staatsmänner; den einzigen Aristeides nimmet er aus, der im Menon, da es sich bloss um die Lehrbarkeit handelte, natürlich ganz auf gleicher Linie mit den Lebrigen behandelt werden durfte; denn aus Erkenntniss handelte er so wenig wie die Andern ${ }^{14}$ ). Läge ein Widerspruch vor - direct kann er nicht vorliegen, weil der Fragepunkt ein anderer ist - so wäre er zu erklären, nicht aus einer vom Gorgias zum Menon milder gewordenen Stimmung, sondern daraus, dass Platon im Gorgias die Sache ernstlicher nimmt und, weil in andrer Absicht, auch mit andern Augen ansieht. Dass von einer Milderung des Urtheils keine Rede sein kann, bestätigt ja doch die Vergleichung späterer Schriften, namentlich des Staates. Man muss woll folgern: da die

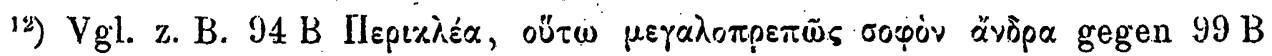

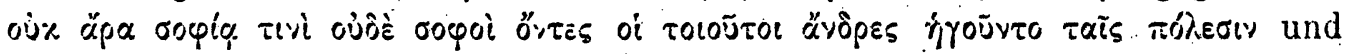

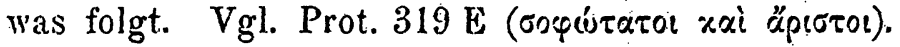

13) Ich übersehe nämlich nicht, dass Platon auch in diesem Endurtheil keirien lebenden Staatsmann als tüchtig anzuerkennen scheint, wie $503 \mathrm{~B}$, $521 \mathrm{D}$.

14) Am wenigsten kann ich in der Auswahl der Namen im Menon eine Zurückbezichung auf den Gorgias erkennen. Perikles war schon in Protagoras, Thukydides und Aristeides im Laches unter demselben Gesichtspunkt behandelt worden; hinzugekommen ist Themistokles. Der Gesichtspunkt des Kallikles (Gorg. $503 \mathrm{C}$ ) ist ein ganz anderer; Aristeides wird von ihm nicht (sondern erșt $526 \mathrm{~B}$ von Sokrates) genannt, Thukydides fehlt ganz. 
Behandlung im Menon übereinstimmt mit der Apologie und dem Protagoras, das schärfere Urtheil des Gorgias dagegen mit unzweifelhaft späteren Schriften, so wird Menon nicht zwischen den Gorgias und diese späteren Schriften, sondern zwischen Protagoras und Gorgias zu setzen sein.

Ich schliesse: sind Protagoras, Laches, Charmides, Menon später geschrieben als die Apologie nebst Kriton; ist insbesondere der Menon, wegen der Anspielung auf das spätere Schicksal des Anytos und der Erwähnung der unrechtlichen Bereicherung des Ismenias, frühstens 395, aber schwerlich auch viel später geschrieben, so erhalten wir für den Gorgias, der alle diese Schriften voraussetzt, zunächst einen terminus post quem. Den terminus ante quem liefert der Theätet, wenn derselbe, wie ich mit Zeller annehme, gegen Ende der $90 \mathrm{er}$ Jahre verfasst ist. Noch zwischen Gorgias und Theätet würde ich den Phädrus setzen aus Gründen, die nur zum kleinsten Theile im Obigen angedeutet werden konnten, übrigens an anderem Orte zu entwickeln sind. Um für Phädrus und Theätet, insbesondere für das von beiden vorausgesetzte wachsende Ausehen des platonischen Kreises Zeit zu lassen, wird man gut thun, den Gorgias möglichst nahe an den Menon heranzurïcken.

Nur wenige Forscher haben dem Gorgias einen wesentlich späteren Termin anweisen wollen. Dass Beziehungen auf die syrakusischen Erlebnisse vorlägen, wie Schleiermacher annahm, wird heute wohl ron Niemand mehr festgehalten, und gar in Isokrates' Rede an Nikokles die "ü̈chste Replik" auf den Gorgias zu erkennen (Teichmüller, Lit. Fehden II, 18f.), wird wohl mainchem Andern ebensorenig wie mir gelingen. 